\title{
Distribution, abundance and host association of two parasitoid species attacking frugivorous drosophilid larvae in central Japan
}

\author{
HideYUKi MITSUI and MASAHITO T. KIMURA* \\ Graduate School of Environmental Earth Science, Hokkaido University, Sapporo, Hokkaido 060-0810, Japan; \\ e-mail:mtk@ees.hokudai.ac.jp
}

Key words. Hymenoptera, Asobara japonica, Ganaspis xanthopoda, parasitoids, drosophilid flies, abundance, distribution, coevolution, host association

\begin{abstract}
In central Japan Ganaspis xanthopoda and Asobara japonica commonly parasitize the larvae of frugivorous drosophilids, mainly in montane forests, and urban environments and small groves, respectively. These two parasitoids start reproduction about one month later than their host drosophilids, probably to avoid searching for hosts when host density is low in early spring. It is likely that the local variation in the abundance of these parasitoids and a temporal refuge for their hosts contribute to the persistence of this parasitoid-host community. The forest species, G. xanthopoda, parasitized at least three Drosophila species that are abundant in forests, supporting the hypothesis that parasitoids are better adapted to attack frequently-encountered host species. This parasitoid did not parasitize drosophilid species that are phylogenetically distantly-related to the three host species or less frequent in forests. Benefits of using such species as host would not exceed the costs of evolving virulence to them. Another parasitoid, A. japonica, parasitized various indigenous and exotic drosophilid species including those that it rarely encountered in the field. It is not clear why this species has such a wide host range.
\end{abstract}

\section{INTRODUCTION}

How the abundance of a species is determined is one of central issues in ecology. Theoretical studies on the dynamics of interaction in parasitoid-host systems suggest that these systems are stable if there is spatial heterogeneity in parasitism or there are refuges for hosts (Nicholson, 1933; Hassell \& May, 1973; Münster-Swendsen \& Nachman, 1978; Hassell et al., 1991; Lei \& Hanski, 1998; Hassell, 2000). However, the interaction is not the only factor affecting the population dynamics, as various environmental factors such as climate, habitat and resource conditions also have large effects. Here, the distribution, abundance and seasonality of parasitoids attacking frugivorous drosophilids in the suburbs of Tokyo were investigated in order to understand how their abundance and distribution are determined. The community studied is composed of two major parasitoids, Ganaspis xanthopoda (Ashmead) (Hymenoptera: Figitidae) and Asobara japonica Belokobylskij (Hymenoptera: Braconidae), and several species of drosophilids (Nishiharu, 1980; Mitsui et al., 2007; Ideo et al., 2008). For this area, the distribution, abundance and seasonality of the drosophilid flies are well documented (Nishiharu, 1980; Beppu, 2000, 2006; Mitsui \& Kimura, 2000a, b; Mitsui et al., 2010), whereas little is known about the ecology of the parasitoids (Mitsui et al., 2007; Ideo et al., 2008).

The distribution and abundance of parasitoids and hosts are important factors in the evolution of host-parasitoid associations (Sasaki \& Godfray, 1999; Lapchin, 2002). If the frequency of encounter does not change temporally and locally, parasitoids specialize on the most frequently encountered host, which evolves resistance to being attacked by these specific parasitoids (Lapchin, 2002). As a consequence an arms-race could operate between parasitoids and hosts, leading to a cyclic change in virulence and resistance (Sasaki \& Godfray, 1999). If the frequency of encounter with antagonists fluctuates between generations, generalist host resistance and partially specialist parasitoid virulence are favoured (Lapchin, 2002). In this paper, it was assessed whether these models explain the association of the present parasitoid and drosophilid species.

\section{MATERIAL AND METHODS}

\section{Parasitoid species}

Asobara japonica in the study areas is parthenogenetic, while G. xanthopoda reproduce sexually (Mitsui et al., 2007; Murata et al., 2009). In a previous paper (Mitsui et al., 2007), the Ganaspis individuals that emerged from $D$. simulans Sturtevant, D. lutescens Okada, D. auraria Peng, D. rufa Kikkawa \& Peng, D. suzukii (Matsumura), Scaptodrosophila coracina (Kikkawa \& Peng) and S. subtilis (Kikkawa \& Peng) were identified as $G$. xanthopoda using morphological characteristics. In a recent study, however, the individuals that emerged from $D$. lutescens did not parasitize $D$. suzukii or $S$. coracina larvae (data presented in this paper), and those that emerged from D. suzukii did not parasitize $D$. lutescens larvae (unpubl. data), suggesting $G$. xanthopoda could consist of at least two species. In this study, the experimental strain of Ganaspis originated from individuals that had emerged from $D$. lutescens. This strain was tentatively assigned to G. xanthopoda, since it was closer to specimens treated as G. xanthopoda by Schilthuizen et al. (1998) in terms of the nucleotide sequence of the second ribosomal internal

\footnotetext{
* Corresponding author
} 


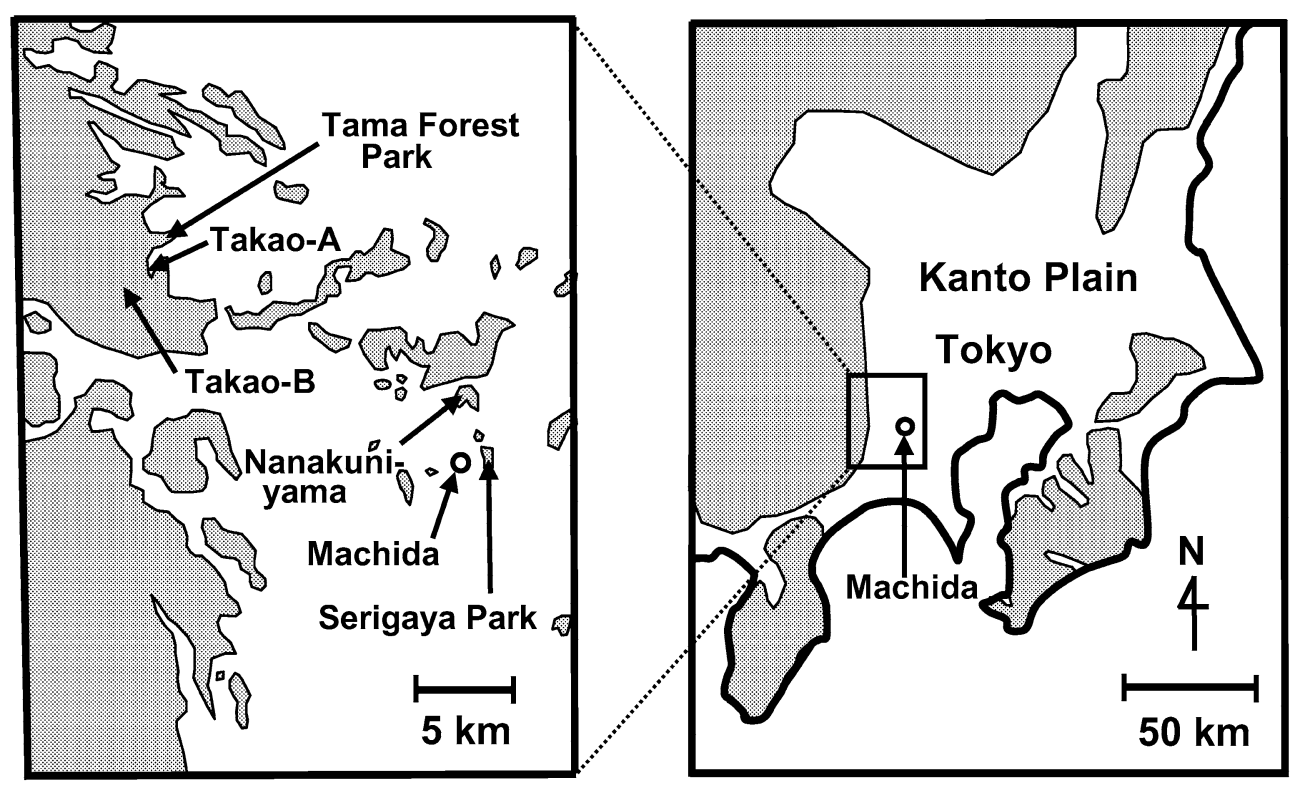

Fig. 1. Locations of the sites studied. Shaded areas indicate forests.

transcribed spacer (ITS2) than to those that emerged from $D$. suzukii (unpubl. data).

\section{Field survey}

Field survey was conducted in the suburbs of Tokyo located on the Kanto Plain (about 17,000 $\mathrm{km}^{2}$ in area). The northern and western sides of the Plain are mountain ranges that are almost covered by forests, and the southern and eastern edges face the sea (Fig. 1). Rural, residential and urban areas spread over the Plain, interspersed with small groves and fragments of forest.

Parasitism was determined at three localities, Machida (one study site), Serigaya Park (two sites) and Nanakuni-yama (two sites), in the center of the Plain, and three localities, Tama Forest Park (two sites), Takao-A (two sites) and Takao-B (three sites), in montane forest regions at or near the western boundary of the Plain (Fig. 1). Table 1 summarizes the environmental conditions and altitudes at these localities, and the frequency and period in a year when the collections were made. Different quantities of banana bait $(1,2,20$ or $30 \mathrm{~g})$ in containers were placed at the study sites, left for one week and then brought back to the laboratory. When the drosophilid larvae in the baits pupated, they were collected, identified to species and whether flies or parasitoids emerged recorded.

The drosophilid species that emerged from the banana baits are only a part of the frugivorous drosophilid fauna. In order to obtain more general information on the frugivorous drosophilid fauna in the area, the drosophilid species breeding in naturallyoccurring fruit at the above localities (Table 1) from spring to autumn in 2005 to 2009 and those breeding in the fruit of Styrax japonicus Siebold \& Zuccarinian (indigenous species) and
Prunus avium L. (cultivated species) fruits in an urban area (Machida) in autumn 1996 were recorded. Fruit was brought to the laboratory, placed in vials with vermiculite and the emergence of drosophilid flies recorded. This survey revealed that the fruit of nineteen species of natural fruits were exploited by drosophilid flies (Mitsui et al., 2010).

\section{Host association}

The experiments used laboratory strains of parasitoids and drosophilids that originated from several females collected in the suburbs of Tokyo in 2006. The drosophilid species used in the experiments are listed in Table 4. The A. japonica and $G$. xanthopoda were reared on D. simulans and D. lutescens, respectively, and the latter on a cornmeal-malt medium. Two or three generations of the hosts and parasitoids were reared in the laboratory $\left(15 \mathrm{~L}: 9 \mathrm{D}\right.$ at $\left.23^{\circ} \mathrm{C}\right)$ before the experiments.

The incidence of parasitism was determined as follows. A number of two- or three-day-old drosophilid larvae were placed with few two- or three-day-old parasitoid females in a Petri dish containing a small amount of rearing medium and observed under a stereoscopic microscope. When drosophilid larvae were parasitized, they were transferred to vials containing food medium and the emergence of parasitoids or drosophilids recorded. For each drosophilid species, about 30 larvae were parasitized by each parasitoid species. The number of parasitoid females used to parasitize one drosophilid species was 5-10 for G. xanthopoda and 2-5 for A. japonica. The experiments were done at $23^{\circ} \mathrm{C}$.

In the above experiments, G. xanthopoda females did not oviposit in some drosophilid species, so the oviposition preferences

TABLE 1. Environmental conditions and altitude (above sea level) at the different localities, and the frequency and period in a year when the collections were made.

\begin{tabular}{lccc}
\hline Locality & Environments & Altitude $(\mathrm{m})$ & Frequency and period in a year when the collections were made \\
\hline Machida & Urban & 50 & 11 times from April to December, 2003 \\
Serigaya Park & Small grove & 50 & 11 times from April to December, 2003 \\
Nanakuni-yama & Fragment of forest & 100 & Every week from March to July, 2005 \\
Tama Forest Park & Edge of montane forests & 200 & Every week from March to June, 2005 \\
Takao-A & Edge of montane forests & 200 & Every week from May to November, 2008 \\
Takao-B & Montane forest & $350-600$ & Every week from May to November, 2008 \\
\hline
\end{tabular}


TABLE 2. Total number of drosophilid pupae collected and numbers of pupae per $30 \mathrm{~g}$ of banana bait, and the proportion of the pupae parasitized by A. japonica $(A j), G$. xanthopoda $(G x)$ and other parasitoid species $(\mathrm{O})$ at the various localities in the suburbs of Tokyo.

\begin{tabular}{|c|c|c|c|c|c|}
\hline & \multicolumn{2}{|c|}{ Pupae collected } & \multicolumn{3}{|c|}{ Parasitoids } \\
\hline & Total & per $30 \mathrm{~g}$ & $A j$ & $G x$ & $\mathrm{O}$ \\
\hline \multicolumn{6}{|c|}{ Machida (garden in urban area) } \\
\hline D. simulans* & 3189 & 63.8 & 0.059 & 0 & 0.010 \\
\hline D. lutescens & 2816 & 56.3 & 0.020 & $<0.001$ & 0.005 \\
\hline D. auraria** & 1048 & 21.0 & 0.006 & 0.006 & 0.014 \\
\hline D. immigrans & 655 & 13.1 & 0 & 0 & 0 \\
\hline Others & 38 & 0.8 & 0 & 0 & 0 \\
\hline \multicolumn{6}{|c|}{ Serigaya park (small grove in urban area) } \\
\hline D. simulans $*$ & 153 & 2.9 & 0.464 & 0 & 0 \\
\hline D. lutescens & 3866 & 72.9 & 0.432 & 0.003 & 0 \\
\hline D. auraria $* *$ & 122 & 2.3 & 0.467 & 0 & 0 \\
\hline D. rufa & 71 & 1.3 & 0.268 & 0 & 0 \\
\hline D. immigrans & 1127 & 21.3 & 0.001 & 0 & 0 \\
\hline Others & 35 & 0.7 & 0 & 0 & 0 \\
\hline \multicolumn{6}{|c|}{ Nanakuni-yama (fragment of forest) } \\
\hline D. lutescens & 2719 & 108.8 & 0.326 & 0.005 & 0.003 \\
\hline D. rufa & 38 & 1.5 & 0.105 & 0 & 0 \\
\hline D. immigrans & 530 & 21.2 & 0 & 0 & 0 \\
\hline S. subtilis & 160 & 6.4 & 0.231 & 0 & 0.031 \\
\hline S. coracina & 161 & 6.4 & 0.019 & 0 & 0.031 \\
\hline Others & 60 & 2.4 & 0.067 & 0 & 0 \\
\hline \multicolumn{6}{|c|}{ Tama forest park (edge of montane forest) } \\
\hline D. lutescens & 5719 & 309.7 & 0.008 & 0.160 & 0.003 \\
\hline D. rufa & 25 & 1.4 & 0.200 & 0.080 & 0 \\
\hline D. bifasciata & 92 & 5.0 & 0.022 & 0 & 0 \\
\hline D. immigrans & 213 & 11.5 & 0 & 0 & 0 \\
\hline S. coracina & 50 & 2.7 & 0 & 0 & 0.180 \\
\hline Others & 51 & 2.7 & 0 & 0 & 0 \\
\hline \multicolumn{6}{|c|}{ Takao-A (edge of montane forest) } \\
\hline D. simulans* & 587 & 11.6 & 0.199 & 0.060 & 0 \\
\hline D. lutescens & 6837 & 134.9 & 0.056 & 0.408 & 0.001 \\
\hline D. rufa & 55 & 1.1 & 0.127 & 0.127 & 0 \\
\hline D. immigrans & 4140 & 81.7 & 0 & 0 & 0 \\
\hline Others & 170 & 3.4 & 0.042 & 0 & 0 \\
\hline \multicolumn{6}{|c|}{ Takao-B (montane forest) } \\
\hline D. simulans* & 169 & 2.3 & 0 & 0 & 0 \\
\hline D. lutescens & 16055 & 215.0 & 0.009 & 0.340 & 0.001 \\
\hline D. rufa & 294 & 3.9 & 0.003 & 0.099 & 0 \\
\hline D. immigrans & 3683 & 49.3 & 0 & 0 & 0 \\
\hline Others & 351 & 4.7 & 0 & 0 & 0 \\
\hline
\end{tabular}

*D. melanogaster may be included; ** D. biauraria Bock et Wheeler or $D$. triauraria Bock et Wheeler may be included.

of this parasitoid were determined. Five two- or three-day old $G$. xanthopoda females were placed with about 30 two- or threeday-old drosophilid larvae in a small Petri dish containing a small amount of rearing medium and left for $4 \mathrm{~h}$ at $23^{\circ} \mathrm{C}$. Then, the drosophilid larvae were dissected under a stereoscopic microscope and the presence or absence of parasitoid eggs recorded.

\section{RESULTS}

\section{Field survey}

Drosophila lutescens was the most abundant drosophilid breeding in banana at all localities, followed by $D$. immigrans Sturtevant (Table 2). In the urban area (Machida), D. simulans was also abundant and D. auraria frequent; the former was almost restricted to urban areas, whereas the latter was also common in small groves. $D$. rufa was commonly recorded in groves and forests.

Drosophila simulans, D. lutescens, D. auraria, D. biauraria, D. rufa, D. suzukii, D. immigrans, D. sternopleuralis Okada and Liodrosophila aerea Okada frequently emerged from the wild, S. japonicus and $P$. avium fruit collected from various locations (Table 3). Among them, D. simulans was almost restricted to urban areas, D. auraria mainly to urban areas and small groves, 


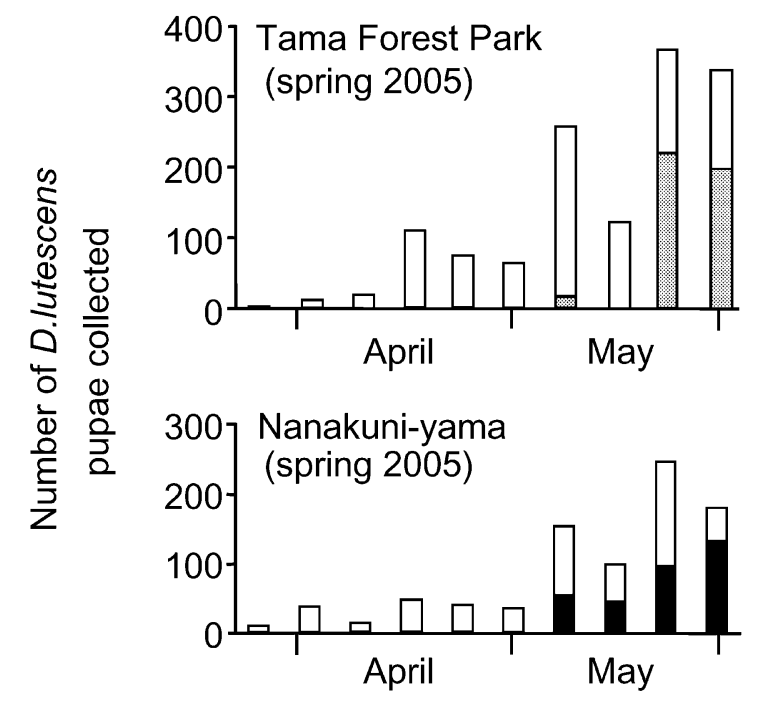

Parasitized by G. xanthopoda

Parasitized by $A$. japonica

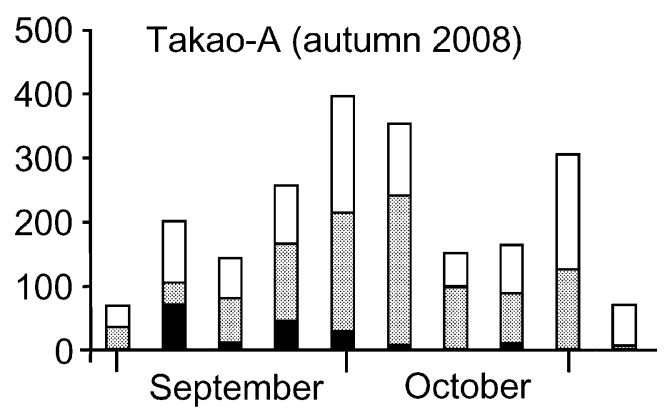

Fig. 2. Number of $D$. lutescens pupae collected per $20 \mathrm{~g}$ of banana bait and the incidence of parasitism at Tama Forest Park (spring 2005), Nanakuni-yama (spring 2005) and Takao-A (autumn 2008).

and D. biauraria, D. rufa and D. suzukii mainly to groves and forests. The other species occurred in various environments.

In the study areas, Asobara japonica and Ganaspis xanthopoda were the dominant parasitoids of the drosophilid larvae that developed in the banana baits placed at the different localities (Table 2). Parasitism by $A$. japonica was higher in the urban area (Machida), a grove (Serigaya Park) and a fragment of forest (Nanakuni-yama) than in montane forest regions (Tama Forest Park, Takao-A and -B), whereas parasitism by G. xanthopoda was higher in montane forest regions. Asobara japonica parasitized various drosophilid species excluding D. immigrans, whereas $G$. xanthopoda mainly parasitized D. lutescens and D. rufa.

Fig. 2 shows the incidence of parasitism of $D$. lutescens at Nanakuni-yama and Tama Forest Park in spring and at Takao-A in autumn. Drosophila lutescens started laying

TABLE 3. Number of drosophilid flies that emerged from naturally-occurring fruit (Nat) collected from various localities and Styrax japonicus and Prunus avium fruit (S\&P) from urban areas. TFP (Tama Forest Park). Environmental conditions at TFP, Takao-A and Takao-B are given in Table 1.

\begin{tabular}{lcccccc}
\hline & \multicolumn{3}{c}{ Urban areas } & Groves & TFP & Takao- \\
\cline { 2 - 6 } & (Nat) & (S\&P) & Takao- \\
(Nat) & (Nat) & A (Nat) & B (Nat) \\
\hline D. simulans & 220 & 1059 & 1 & 0 & 1 & 0 \\
D. lutescens & 46 & 39 & 256 & 41 & 53 & 190 \\
D. auraria & 0 & 68 & 9 & 49 & 27 & 0 \\
D. biauraria & 0 & 0 & 2 & 86 & 3 & 21 \\
D. triauraria & 0 & 15 & 0 & 0 & 0 & 0 \\
D. rufa & 0 & 36 & 159 & 86 & 7 & 195 \\
D. suzukii & 2 & 7 & 251 & 381 & 6 & 377 \\
D. sternopleuralis & 0 & 152 & 41 & 32 & 0 & 801 \\
D. bizonata & 0 & 4 & 2 & 0 & 0 & 1 \\
D. immigrans & 0 & 95 & 85 & 0 & 8 & 48 \\
L. aerea & 0 & 20 & 5 & 57 & 0 & 394 \\
Others & 19 & 12 & 42 & 31 & 43 & 26 \\
\hline Total & 287 & 1507 & 853 & 763 & 148 & 2053 \\
\hline
\end{tabular}

eggs in late March, but parasitism by $A$. japonica and $G$. xanthopoda occurred about one month later in early May. This delay in the onset of parasitism was also recorded for A. japonica in Serigaya Park (data not shown).

\section{Host association}

In the oviposition preference test, G. xanthopoda females oviposited in D. lutescens, D. biauraria, D. rufa, $D$. auraria and $D$. triauraria larvae more frequently than in those of D. simulans and D. suzukii, and it did not oviposit in those of D. sternopleuralis, D. bizonata Kikkawa $\&$ Peng or $S$. coracina (Table 4).

In terms of emergence Ganaspis xanthopoda more successfully parasitized $D$. lutescens, D. biauraria and $D$. rufa than D. triauraria and D. simulans, in which this parasitoid suffered a rather high mortality (Table 5). This parasitoid did not survive in D. auraria larvae. Asobara japonica successfully parasitized all the drosophilid species included in this study (Table 5).

\section{DISCUSSION}

\section{Distribution, abundance and seasonality}

In central Japan the most important parasitoids attacking frugivorous drosophilids are Ganaspis xanthopoda and Asobara japonica (Mitsui et al., 2007). They

TABLE 4. Percentages of drosophilid larvae parasitized by $G$. xanthopoda in the oviposition preference test.

\begin{tabular}{lcc}
\hline & N & Percentage parasitized \\
\hline D. simulans & 30 & 16.7 \\
D. lutescens & 30 & 93.3 \\
D. auraria & 30 & 60.0 \\
D. biauraria & 30 & 86.7 \\
D. triauraria & 30 & 50.0 \\
D. rufa & 29 & 73.3 \\
D. suzukii & 28 & 3.3 \\
D. sternopleuralis & 29 & 0 \\
D. bizonata & 29 & 0 \\
S. coracina & 20 & 0 \\
\hline
\end{tabular}


TABLE 5. Number (percentage) of drosophilid larvae from which flies or parasitoids, or neither emerged, when they were parasitized by A. japonica or G. xanthopoda.

\begin{tabular}{lccccccccc}
\hline & \multicolumn{4}{c}{ A. japonica } & & \multicolumn{3}{c}{ G. xanthopoda } \\
\cline { 2 - 3 } & $\begin{array}{c}\text { No. of larvae } \\
\text { parasitized }\end{array}$ & $\begin{array}{c}\text { Fly } \\
\text { emerged }\end{array}$ & $\begin{array}{c}\text { Parasitoid } \\
\text { emerged }\end{array}$ & $\begin{array}{c}\text { Neither fly or } \\
\text { parasitoid emerged }\end{array}$ & $\begin{array}{c}\text { No. of larvae } \\
\text { parasitized }\end{array}$ & $\begin{array}{c}\text { Fly } \\
\text { emerged }\end{array}$ & $\begin{array}{c}\text { Parasitoid } \\
\text { emerged }\end{array}$ & $\begin{array}{c}\text { Neither fly or } \\
\text { parasitoid emerged }\end{array}$ \\
\hline D. simulans & 30 & $3(10.0)$ & $21(70.0)$ & $6(20.0)$ & & 27 & $2(7.4)$ & $6(22.2)$ & $19(72.0)$ \\
D. lutescens & 30 & $2(6.7)$ & $22(73.3)$ & $6(20.0)$ & & 33 & $0(0)$ & $21(63.6)$ & $12(36.4)$ \\
D. auraria & 33 & $1(3.0)$ & $26(86.7)$ & $6(18.2)$ & & 20 & $3(15.0)$ & $0(0)$ & $17(85.0)$ \\
D. biauraria & 30 & $1(3.3)$ & $18(60.0)$ & $11(36.7)$ & & 34 & $2(5.9)$ & $22(66.6)$ & $10(29.4)$ \\
D. triauraria & 36 & $0(0)$ & $26(72.2)$ & $10(27.8)$ & & 31 & $0(0)$ & $10(32.3)$ & $21(67.7)$ \\
D. rufa & 32 & $1(3.1)$ & $22(68.8)$ & $9(28.1)$ & & 30 & $3(10.0)$ & $13(43.3)$ & $14(46.7)$ \\
D. suzukii & 31 & $0(0)$ & $21(67.7)$ & $10(32.3)$ & & - & & & \\
D. sternopleuralis & 30 & $0(0)$ & $21(70.0)$ & $9(30.0)$ & & - & & & \\
D. bizonata & 31 & $0(0)$ & $27(87.1)$ & $4(12.9)$ & & - & & & \\
S. coracina & 30 & $1(3.3)$ & $19(63.3)$ & $10(33.3)$ & & - & & \\
\hline
\end{tabular}

differed in their distributions with the former more abundant in montane forest regions and the latter in urban areas, small groves and fragments of forest. Similar differences in the distributions of the drosophilid flies are also recorded; D. simulans is almost only recorded in urban areas, D. auraria mainly in urban areas and groves, and D. biauraria, D. rufa and D. suzukii mainly in groves and forests (Kurokawa, 1967; Kawanishi \& Watanabe, 1977; Nishiharu, 1980; Kimura, 1987; Mitsui \& Kimura, $2000 \mathrm{a}, \mathrm{b})$. It is unknown what determines their distributions. They may select habitats in response to temperature, humidity or light regimes that differ in urban, open and wooded regions. If this is the case then why do they prefer different habitats? For G. xanthopoda, the distribution of hosts may affect its habitat selection; this parasitoid is recorded most abundantly in forests that are the habitat of its hosts, D. lutescens, D. rufa and D. biauraria (Kurokawa, 1967; Nishiharu, 1980; Kimura, 1987; Mitsui \& Kimura, 2000a, b). However, A. japonica was rarely recorded in montane forests, although it can parasitize a wide variety of drosophilid flies including forestinhabiting species. For this species, its colonization history may influence its habitat selection. The populations of this species on the main islands of Japan are parthenogenetic, but differ little in the nucleotide sequence of the cytochrome oxidase subunit I (COI) gene from the supposed ancestral sexually-reproducing subtropical populations (Murata et al., 2009), which indicates this species may have only rather recently colonized the main islands. Thus, this species may not have had enough time to adapt to the natural environments (forests) on the main islands and hence its occurrence only in urban areas, like some exotic species of Drosophila (e.g. D. simulans).

The two parasitoids started parasitism in early May, about a month later than the appearance of host larvae, probably to avoid searching for hosts when host density is low in early spring. Thus, the drosophilids have a temporal refuge from parasitism in early spring.

It is suggested that spatial heterogeneity in parasitism or a temporal refuge for hosts is required for the persistence of parasitoid and host populations (Nicholson, 1933; Hassell \& May, 1973; Münster-Swendsen \& Nach- man, 1978; Hassell et al., 1991; Hassell, 2000). In the present case, in fact, there is considerable spatial variation in the incidence of parasitism and temporal refuges for the host drosophilids. Moreover, the parasitoids used a number of Drosophila species as hosts, a situation that also contributes to the stabilization of parasitoid-host systems (Murdoch \& Oaten, 1975; Southwood \& Comins, 1976; Hassell \& May, 1986).

\section{Evolution of host associations}

Lapchin (2002) predicted that parasitoids will become better adapted to exploit frequently-encountered host species. In fact, the forest-inhabiting parasitoid, G. xanthopoda, is well adapted to exploit at least $D$. lutescens, $D$. rufa and $D$. biauraria, which are abundant in forests. In addition, these host species show temporal and spatial variation in abundance (Kurokawa, 1967; Kawanishi \& Watanabe, 1977; Kimura, 1987; Beppu, 2000, 2006; Mitsui \& Kimura, 2000a, b; Mitsui et al., 2010), which would prevent specialization on a single drosophilid species (Lapchin, 2002).

Ganaspis xanthopoda rarely, or did not, oviposit in $D$. suzukii or D. sternopleuralis which were abundant in fruit in forest areas. Further, field data suggest that this wasp does not use $D$. immigrans, an abundant species not only in urban areas but also in montane forests. The reason for this is unknown, but there are hypotheses. Drosophila suzukii differs in its ecology from the three host species; it oviposits in fresh fruit before it falls from the trees, whereas the host species oviposit in fermenting fruit on the ground (Mitsui et al., 2006). For parasitoids, it may be costly to search for hosts both in the canopy of trees and on the ground. On the other hand, D. sternopleuralis and D. immigrans belong to the subgenus Drosophila (Okada \& Kurokawa, 1957), whereas the three host species and D. suzukii belong to the subgenus Sophophora. It may be costly for parasitoids to adapt to taxonomically diverse species.

In contrast to the above drosophilid species, D. auraria, $D$. bizonata and $S$. coracina are uncommon in fruits in forest where $G$. xanthopoda is abundant; $D$. auraria inhabits urban areas and open lands, D. bizonata mainly breeds in mushrooms and $S$. coracina frequently breeds 
in tree sap and mushrooms (Kurokawa, 1967; Kimura et al., 1977; Kawanishi \& Watanabe, 1977; Nishiharu, 1980; Kimura, 1987; Mitsui \& Kimura, 2000a, b). This may be the reason why $G$. xanthopoda does not parasitize these drosophilids. On the other hand, this wasp is able to successfully parasitize $D$. simulans (urban species) and $D$. triauraria (open land species) to some extent, although it seldom encounters them. Its ability to attack these species may be a by-product of its ability to parasitize the major host species, e.g., D. biauraria or D. lutescens.

Another parasitoid, Asobara japonica, parasitizes a variety of drosophilid species occurring in this area (Ideo et al., 2008). It is not clear why it has such a wide host range. As referred to previously, this wasp is assumed to have colonized the main islands of Japan rather recently (Murata et al., 2009) and to have had only a short history of interactions with the drosophilid populations there. This may indicate that its ability to parasitize these drosophilids is a by-product of its ability to parasitize the major host species in subtropical areas where it has originated (Murata et al., 2009). On the other hand, some subtropical Drosophila species have evolved resistance to this parasitoid (Ideo et al., 2008; Furihata \& Kimura, 2009; Murata et al., 2009), suggesting co-evolutionary interactions.

ACKNOWLEDGEMENTS. This work was supported by a Grant-in-Aid from the Ministry of Education, Science, Sports and Culture of Japan (No. 17570010).

\section{REFERENCES}

BEPPU K. 2000: Faunal and ecological surveys on drosophilid flies in the Imperial Palace, Tokyo. Mem. Nat. Sci. Mus. 36: 409-435.

BEppu K. 2006: Seasonal change of drosophilid assemblage and adult age structure of the common drosophilid species in the Imperial Palace Grounds, Tokyo. Mem. Nat. Sci. Mus. 43: 295-334 [in Japanese with English abstr.].

Furihata S.X. \& KimURA M.T. 2009: Effects of Asobara japonica venom on larval survival of host and nonhost Drosophila species. Physiol. Entomol. 34: 292-295.

Hassell M.P. 2000: Host-parasitoid population dynamics. J. Anim. Ecol. 69: 543-566.

Hassell M.P. \& May R.M. 1973: Stability in insect hostparasite models. J. Anim. Ecol. 42: 693-726.

Hassell M.P. \& MAY R.M. 1986: Generalist and specialist natural enemies in insect predator-prey interactions. J. Anim. Ecol. 55: 923-940.

Hassell M.P., Pacala S.W., May R.M. \& Chesson P.L. 1991: The persistence of host-parasitoid associations in patchy environments. I. A general criterion. Am. Nat. 138: 568-583.

Ideo S., Watada M., Mitsui H. \& Kimura M.T. 2008: Host range of Asobara japonica (Hymenoptera: Braconidae), a larval parasitoid of drosophilid flies. Entomol. Sci. 11: 1-6.

KaWANishi M. \& Watanabe T.K. 1977: Ecological factors controlling the coexistence of the sibling species Drosophila simulans and D. melanogaster. Jpn. J. Ecol. 27: 279-283.

Kimura M.T. 1987: Habitat differentiation and speciation in the Drosophila auraria species-complex (Diptera: Drosophilidae). Kontyû 55: 429-436.
Kimura M.T., Toda M.J., Beppu K. \& Watabe H. 1977: Breeding sites of drosophilid flies in and near Sapporo, northern Japan, with supplementary notes on adult feeding habits. Kontyû 45: 571-582.

Kunokawa H. 1967: Population genetics of three races of Drosophila auraria Peng. III. Geographical and ecological distribution of the races, $\mathrm{A}, \mathrm{B}$, and $\mathrm{C}$, with special regard to its speciation. Jpn. J. Genet. 42: 109-119.

LAPCHIN L. 2002: Host-parasitoid association and diffuse coevolution: when to be a generalist? Am. Nat. 160: 245-254.

Lei G.C. \& Hanski I. 1998: Spatial dynamics of competing specialist parasitoids in host metapopulation. J. Anim. Ecol. 67: 422-433.

Mitsui H. \& Kimura M.T. 2000a: Food preference of drosophilid flies in domestic and forest areas of central Japan. Entomol. Sci. 3: 285-289.

Mitsui H. \& Kimura M.T. 2000b: Coexistence of drosophilid flies: aggregation, patch size diversity and parasitism. Ecol. Res. 15: 95-100.

Mitsui H., TAKahashi K.H. \& KimURa M.T. 2006: Spatial distributions and clutch sizes of Drosophila species ovipositing on cherry fruits of different stages. Popul. Ecol. 48: 233-237.

Mitsui H., van Achterberg K., Nordlander G. \& Kimura M.T. 2007: Geographical distributions and host associations of larval parasitoids of frugivorous Drosophilidae in Japan. $J$. Nat. Hist. 41: 1731-1738.

Mitsui H., Beppu K. \& Kimura M.T. 2010: Seasonal life cycles and resource uses of flower- and fruit-feeding drosophilid flies (Diptera: Drosophilidae) in central Japan. Entomol. Sci. 13: $60-67$.

Münster-Swendsen M. \& Nachman G. 1978: Asynchrony in insect-host parasite interactions and its effect on stability, studied by a simulation model. J. Anim. Ecol. 50: 855-865.

Murata Y., Ideo S., Watada M., Mitsui H. \& Kimura M.T. 2009: Genetic and physiological variation among sexual and parthenogenetic populations of Asobara japonica (Hymenoptera: Braconidae), a larval parasitoid of drosophilid flies. Eur. J. Entomol. 106: 171-178.

Murdoch W.W. \& OAten A. 1975: Predation and population stability. Adv. Ecol. Res. 9: 2-131.

Nicholson A.J. 1933: The balance of animal populations. $J$. Anim. Ecol. 2: 131-178.

NishiHaru S. 1980: A Study of Ecology and Evolution of Drosophilid Flies with Special Regard to Imaginal and Larval Feeding Habits and Seasonal Population Fluctuations. Doctor of Science Thesis, Tokyo Metropolitan University, Tokyo.

OKada T. \& KuroKawa H. 1957: New or little known species of Drosophilidae of Japan (Diptera). Kontyû 25: 1-12.

SASAKI A. \& Godfray H.C.J. 1999: A model for the coevolution of resistance and virulence in coupled host-parasitoid interactions. Proc. R. Soc. Lond. (B) 266: 455-463.

Schilthuizen M., Nordlander G., Stouthamer R. \& van AlPhEN J.J.M. 1998: Morphological and molecular phylogenetics in the genus Leptopilina (Hymenoptera: Cynipoidea: Eucoilidae). Syst. Entomol. 23: 253-264.

Southwood T.R.E. \& Comins H.N. 1976: A synoptic population model. J. Anim. Ecol. 45: 949-965.

Received December 8, 2009; revised and accepted April 8, 2010 